\title{
The effectiveness of daclatasvir based therapy in European patients with chronic hepatitis $C$ and advanced liver disease
}

\author{
Jim Young ${ }^{1 *}$, Nina Weis ${ }^{2}$, Harald Hofer ${ }^{3}$, William Irving ${ }^{4}$, Ola Weiland ${ }^{5}$, Emiliano Giostra ${ }^{6}$, Juan Manuel Pascasio ${ }^{7}$, \\ Lluis Castells ${ }^{8}$, Martin Prieto ${ }^{9}$, Roelien Postema ${ }^{10}$, Cinira Lefevre ${ }^{11}$, David Evans ${ }^{11}$, Heiner C. Bucher ${ }^{1}$ \\ and Jose Luis Calleja ${ }^{12}$
}

\begin{abstract}
Background: There is limited evidence for the effectiveness of daclatasvir in patients whose hepatitis $C$ threatens their life expectancy. The Named Patient Program in Europe included patients with advanced chronic hepatitis C, a life expectancy of less than 12 months and no other treatment options.

Methods: A retrospective multi-country cohort of patients with chronic hepatitis $C$ who received daclatasvir as part of the Named Patient Program in Austria, Denmark, Spain, Sweden, Switzerland and the United Kingdom. Treatment response was defined as a sustained virologic response (unquantifiable hepatitis C RNA) at 12 weeks post treatment. We summarised the characteristics of the patients in this cohort and estimated the rate of sustained virologic response for patients receiving daclatasvir and sofosbuvir with or without ribavirin using hierarchical Bayesian modelling.

Results: The 249 patients included had a median age of 56 years; most were male (78\%), hepatitis C genotype 1 (75\%), treatment experienced (65\%) and with decompensated cirrhosis (59\%). Many had had a liver transplant before receiving daclatasvir (40\%). Of the 249 patients, 242 patients received daclatasvir and sofosbuvir and either reached 12 weeks post treatment or died during $(n=9)$ or after treatment $(n=4)$ or were lost to follow up during treatment $(n=1)$. The estimated rate of sustained virologic response at 12 weeks post treatment was $87 \%$ (95\% credible interval 75 to 94\%) for previously treated genotype 1 patients with decompensated cirrhosis.
\end{abstract}

Conclusions: Daclatasvir with sofosbuvir is an effective treatment in clinical practice for hepatitis $C$ genotype 1 patients with decompensated cirrhosis.

Keywords: Daclatasvir, Direct-acting antivirals, Effectiveness, Hepatitis C, Sofosbuvir

\section{Background}

Chronic hepatitis $\mathrm{C}$ often leads to cirrhosis and hepatocellular carcinoma [1]. While the prevalence of hepatitis $\mathrm{C}$ virus $(\mathrm{HCV})$ infection is expected to decline worldwide, the number of individuals with end stage liver disease is projected to rise [2] and with it, the cost of healthcare for these individuals [3]. Earlier standard treatment with subcutaneous pegylated interferon alfa and oral ribavirin achieved sustained virologic response (SVR) rates of only

\footnotetext{
*Correspondence: james.young@usb.ch

'Basel Institute for Clinical Epidemiology and Biostatistics, University Hospital Basel, Spitalstrasse 12, CH-4031 Basel, Switzerland

Full list of author information is available at the end of the article
}

$50 \%$ or less in patients infected with HCV genotype 1 [4], although SVR rates were as high as 60 to $80 \%$ in genotypes 2,3 and $6[4,5]$. In addition, this treatment caused potentially serious side effects and was therefore contraindicated in patients with decompensated cirrhosis [4].

Daclatasvir, a HCV NS5A replication complex inhibitor, has shown encouraging results in clinical trials across multiple genotypes, both in interferon-based regimens and in all oral regimens without interferon [6, 7]. The combination of daclatasvir and sofosbuvir, a nucleotide analogue $\mathrm{HCV}$ NS5B polymerase inhibitor, has shown high rates of SVR in patients with $\mathrm{HCV}$ genotype $1[8,9]$. However there is limited evidence for the effectiveness of this treatment 
combination in patients whose advanced cirrhosis threatens their life expectancy [10-12]. There is also uncertainty about the optimal duration of treatment and whether ribavirin should be added to the combination [10-12].

A Named Patient Programme (NPP) for daclatasvir in Europe included patients from 2012 to 2015 with advanced chronic HCV, a life expectancy of less than 12 months and no other treatment options. Here we summarise the characteristics of the patients in this programme and estimate their rate of SVR when given daclatasvir in combination with sofosbuvir.

\section{Methods}

\section{Patients}

The first use of daclatasvir in Europe outside clinical trials was via Bristol-Myers Squibb's (BMS) early access programmes. The NPP was one of these programmes. Patients were eligible to participate in the NPP if according to their physician: (1) the patient was an adult with chronic hepatitis $C$ (regardless of genotype) at high risk of decompensation or death within 12 months; and (2) there were no satisfactory alternative treatments options for the patient and available treatment options had been exhausted; and (3) the patient was ineligible to participate in a clinical trial where his or her infection might be treated satisfactorily or there was no ongoing clinical trial in the patient's country of residence.

Collaborative agreements were later signed between $\mathrm{BMS}$ and $\mathrm{HCV}$ registries or healthcare institutions with HCV databases in six European countries. These agreements allowed the transfer of individual patient data for patients in a NPP to a third party, the Basel Institute for Clinical Epidemiology and Biostatistics, for analysis. BMS did not have any access to individual patient data. The institutions providing data were: the Department of Internal Medicine III, at the Medical University of Vienna (Austria); eight hospitals in the Danish Database for Hepatitis B and C (Denmark); the Hepa C registry (Spain); the Karolinska Institutet, at Karolinska University Hospital Huddinge (Sweden); the University Hospital Geneva (Switzerland); and seven hospitals in the HCV Research UK registry (United Kingdom). All HCV registries or databases used in this study contained data prospectively collected from adult patients who had given informed consent. We planned to also include data from Italy but agreements with suitable institutions have yet to be signed.

To be eligible for this retrospective cohort study, patients had to start any daclatasvir containing regimen under a NPP (and not under any other early access programme) and have data in one of the above HCV databases. Patients were recruited from December 2012 until August 2014 when daclatasvir was approved for use in Europe but the exact dates varied between countries because in some countries, the NPP was replaced by another early access program.

\section{Statistical methods}

The primary objective of this study was to estimate the effectiveness of daclatasvir in combination with either sofosbuvir or simeprevir as measured by the rate of sustained virologic response at 12 weeks after the end of treatment (SVR12). However only a single patient received daclatasvir with simeprevir, so our analyses were restricted to patients receiving daclatasvir and sofosbuvir, with or without ribavirin. SVR12 was defined as HCV RNA either undetectable or below the assay's lower limit of quantification.

Secondary objectives were to summarise the demographic and clinical characteristics of patients receiving daclatasvir and to assess effectiveness using other measures. Other measures of effectiveness available from all registries included the virologic response at the end of treatment and the sustained virologic response at four weeks after the end of treatment (SVR4).

The protocol (ClinicalTrials.gov NCT02531269) specified an intent-to-treat analysis such that failure to achieve a SVR was assumed if a patient died or was lost to follow up, or if a response was missing and sufficient time had elapsed since the patient finished treatment for that response to be measureable. There was an appreciable rate of mortality in these patients. As a consequence, a decision was made prior to any modelling to add an as-treated analysis in which patients with no recorded response were excluded (either because of death, loss to follow up or a missing response). In both intent-to-treat and as-treated analyses, a SVR at four or 12 weeks was assumed where a response was missing but the patient had a SVR at a later date.

SVR rates were estimated using hierarchical Bayesian modelling because small samples were anticipated from most databases. Each SVR rate was estimated under four models of increasing complexity. In all models we assumed responses were binomially distributed with a different rate for each database and that rates for each database were normally distributed. The first model was without covariates and with an uninformative prior for between database variability; the second model included covariates-for genotype, prior treatment and cirrhosis when starting the current treatment-so that data from each database could be considered exchangeable [13]. The third and fourth models were a repeat of the first two but with a weakly informative prior for between database variability. Models with covariates provide estimates of the SVR rate in a group of reference patients (here $\mathrm{HCV}$ genotype 1 patients, previously treated but now with decompensated cirrhosis); the model can then be used to estimate SVR rates in other covariate subgroups 
(such as previously treated genotype 1 patients with stable or no cirrhosis).

With a small number of databases, our uninformative prior for between database variability-a uniform distribution on the standard deviation of the between database variability-could lead to 'under-pooling' of data [13]. Therefore, in the last two models, we used a weakly informative half-Cauchy prior with its scale parameter set at the prior standard deviation [13] assuming that the lowest and highest SVR rates between databases would be covered with $95 \%$ probability by a range of odds ratios from $1 / 4$ to 4 relative to the average across all databases.

Adding covariates essentially accounts for what could be material differences in patient mix between databases. Weakly informative priors were used for this adjustment: distributions where 'the percentiles of the prior distribution would be viewed as at least reasonable if not liberally inclusive by all those working in the research topic' [14]. Weakly informative priors restrict covariate effects to a range of values that is clinically sensible, ruling out extreme values that no knowledgeable clinician would find plausible [15]. The effects of genotype 3 and of genotypes other than 1 or 3 were both assumed to be of 'uncertain direction' (prior odds ratio (OR) 1.0, 95\% credible interval (CI) 0.25 to 4.0 ); no prior treatment was assumed to be 'possibly beneficial' (prior OR 1.5, 95\% CI 0.38 to 6.0); and no or stable cirrhosis was assumed to be 'probably beneficial' (prior OR 2.0, 95\% CI 0.5 to 8.0$)[16,17]$. There is clinical interest in the value of including ribavirin in treatment combinations but limited information [18, 19]. Given adequate data were collected on the use of daclatasvir and sofosbuvir both with and without ribavirin, the fourth model was extended to include an additional covariate for ribavirin use, with its effect assumed to be of 'uncertain direction' (prior OR 1.0, 95\% CI 0.25 to 4.0 ).

We report estimated SVR rates and their 95\% CI from each model. Fitting a sequence of models allowed us to check that as models became more complex, estimates varied between models in a logical fashion. However the most complex fourth model seems the most appropriate because it better meets the assumption of exchangeability between databases and gives rise to greater pooling of data across databases. Exchangeability implies that each database is independent of the others and, after adjusting for covariates, contains identically distributed data [13]. Models were fit using R 3.1.0, R2WinBUGS 2.1-20 and WinBUGS 1.4.3.

\section{Results}

\section{Patients}

Of the 249 NPP patients identified in the six databases, 246 patients started daclatasvir and sofosbuvir (Fig. 1) with or without ribavirin (65 and 181 patients respectively). At the time of analysis, four of the 246 patients had not been followed for at least 12 weeks after the end of treatment, so that the SVR12 intent-to-treat analysis was of 242 patients. Of these 242 patients, 13 died prior to 12 weeks after treatment, one was lost to follow up during treatment and a response was missing for three patients so that the SVR12 as-treated analysis was of 225 patients. Of the 14 patients that died or were lost to follow up prior to 12 weeks after treatment, 13 had decompensated cirrhosis. The remaining patient received a second liver transplant during treatment and died before completing treatment.

Most of the 249 NPP patients were male (78\%), with a median age of 56 years (Table 1). Most had HCV genotype 1 (75\%), were treatment experienced (65\%), had advanced fibrosis or cirrhosis (82\% F3-F4) and decompensated cirrhosis (59\%) and many had had a liver transplant (40\%). Those given ribavirin were less likely to have decompensated cirrhosis (55\%) and more likely to have had a liver transplant $(44 \%)$ than those not given ribavirin $(61 \%$ and $38 \%$ respectively). Liver transplantation during treatment was reported for 27 patients (11\%); 21 of these patients had decompensated cirrhosis.

For the 246 patients starting daclatasvir and sofosbuvir with or without ribavirin, the planned treatment duration was 24 weeks for all but three patients-these three patients were to receive 8,12 , and 20 weeks of treatment. Among the 243 patients that were to receive 24 weeks of treatment, the actual treatment duration was known for 218 patients: 187 (86\%) were treated for between 23 and 25 weeks, 16 (7\%) were treated for fewer than 23 weeks and 15 (7\%) were treated for more than 25 weeks. Where known, the median duration of treatment was the same for those treated with and without ribavirin: 168 days (interquartile range 168 to 168) for those treated with ribavirin and 168 days (interquartile range 167 to 169) for those treated without ribavirin.

\section{Observed effectiveness}

Of the 234 patients that completed treatment, an end of treatment response was missing for 15 patients; otherwise all patients had an end of treatment virologic response. 92\% of intent-to-treat patients and $97 \%$ of as-treated patients had a SVR4; $90 \%$ of intent-to-treat patients and 97\% of as-treated zpatients had a SVR12 (Table 2). Rates of SVR4 and SVR12 varied between countries, from 82 to $100 \%$ : this variability in response rates between countries was expected because in all countries except Spain, response rates were estimated from fewer than 30 patients.

Of the six patients that failed to achieve a SVR12, all had decompensated cirrhosis, five were treatment experienced and none received ribavirin (Table 3). For one patient, the duration of treatment was not known and the end of treatment response was missing; the other five all received at least 20 weeks of treatment and had 


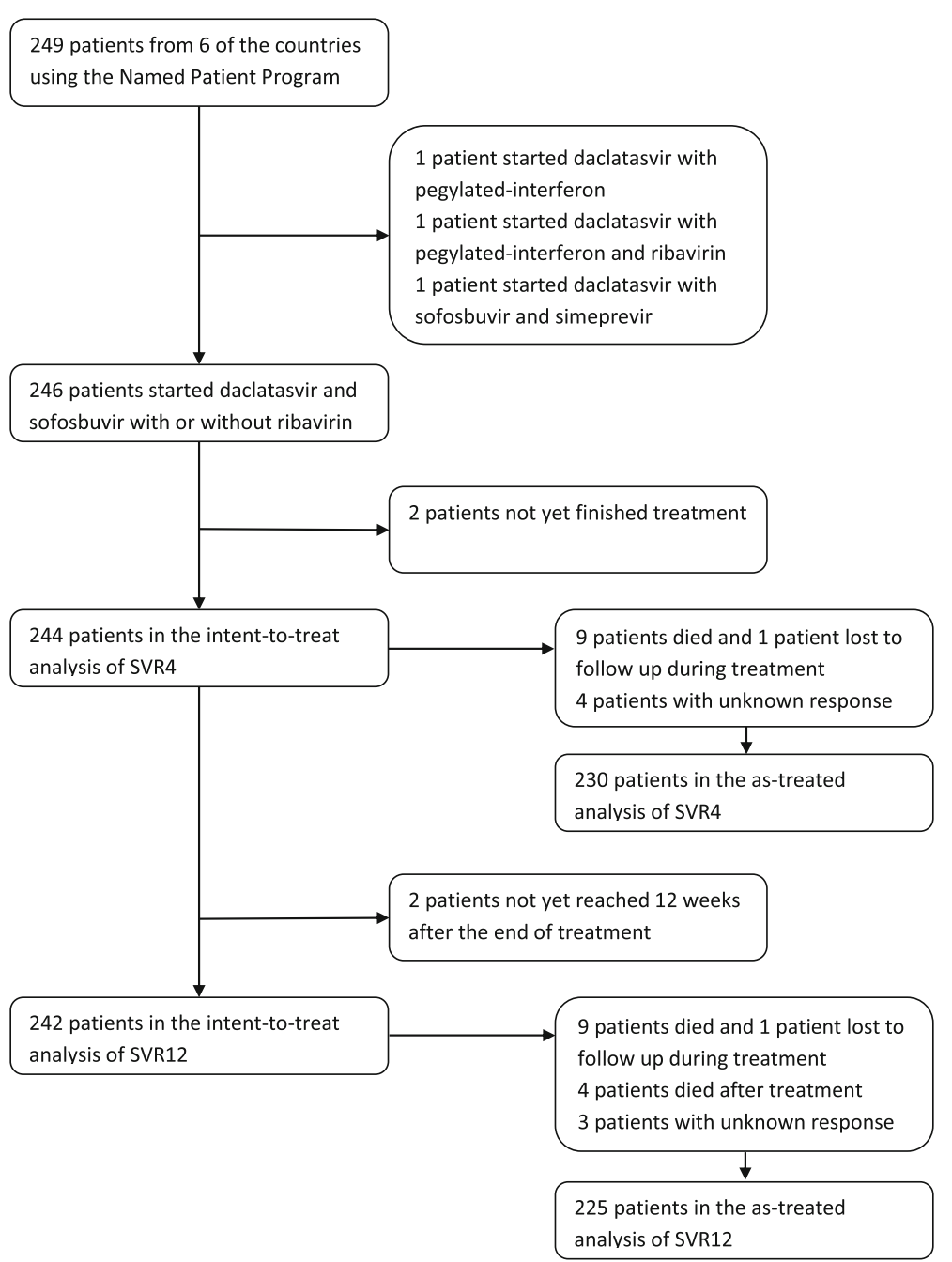

Fig. 1 Patient flow. Patients included in intent-to-treat and as-treated analyses of sustained virologic response at four weeks after the end of treatment (SVR4) and at 12 weeks after the end of treatment (SVR12)

an end of treatment virologic response. Four of the five then had detectable viremia within 4 weeks after the end of treatment; the remaining patient had detectable viremia between 4 and 12 weeks after the end of treatment. Of the four patients with detectable viremia within 4 weeks: one was found to have $\mathrm{Y} 93 \mathrm{H}$ resistance-associated variant (RAV) $\mathrm{HCV}$; one had both $\mathrm{Y} 93 \mathrm{H}$ and Q30H RAV $\mathrm{HCV}$; the virus could not be sequenced for one patient but the patient was successfully re-treated with ledipasvir, sofosbuvir and ribavirin; the fourth patient was found to have L31M, L31I and Y93H RAV HCV but was also successfully re-treated with ledipasvir, sofosbuvir and ribavirin. The remaining patient with detectable viremia between 4 and 12 weeks was genotype 3a and was found to have Y93H RAV HCV.

\section{Estimated effectiveness}

Estimates from the fourth hierarchical Bayesian model are shown for patients with HCV genotype 1, previously treated and now with decompensated cirrhosis (Table 4). These patients were selected as the reference group because they were typical of those treated in the NPP. For such patients, the estimated rate of SVR4 was $89 \%$ (95\% CI 76 to 96\%) and 96\% (95\% CI 86 to 100\%) in intent-to-treat and as-treated analyses respectively; the estimated rate of SVR12 was $87 \%$ (95\% CI 75 to $94 \%$ ) and $97 \%$ (95\% CI 89 to 100\%) in intent-to-treat and astreated analyses respectively. Estimates from all four models are shown in Additional file 1: Tables S1 to S4.

The model provides estimates of the association between virologic response and covariates in the form of posterior OR relative to the response in reference patients. In the SVR12 intent-to-treat analysis, these estimates were: genotype 3, OR 0.69 (95\% CI 0.25 to 1.54); other genotypes OR 1.06 (95\% CI 0.32 to 2.77); no or stable cirrhosis, OR 6.73 (95\% CI 2.42 to 16.1); no prior treatment, OR 0.92 (95\% CI 0.39 to 1.89 ). Extending this model so that it includes an 
Table 1 Patient characteristics when starting hepatitis C (HCV) treatment with daclatasvir $(n=249)$

\begin{tabular}{|c|c|c|c|c|c|c|}
\hline \multirow[t]{3}{*}{ Characteristics } & \multirow{3}{*}{$\begin{array}{l}\text { All } \\
n=249\end{array}$} & \multicolumn{3}{|c|}{ Genotype } & \multicolumn{2}{|c|}{ Ribavirin } \\
\hline & & $1^{\mathrm{a}}$ & $3^{b}$ & Other $^{\mathrm{c}}$ & With & Without \\
\hline & & $n=187$ & $n=40$ & $n=22$ & $n=66$ & $n=183$ \\
\hline Age, median (years) & 56 & 56 & 55 & 55 & 55 & 57 \\
\hline Male (\%) & 78 & 75 & 85 & 91 & 85 & 76 \\
\hline HIV co-infection (\%) & 10 & 8 & 15 & 14 & 11 & 9 \\
\hline Prior HCV treatment (\%) & 65 & 69 & 48 & 64 & 65 & 65 \\
\hline \multicolumn{7}{|l|}{ Cirrhosis (\%) } \\
\hline - No & 14 & 16 & 5 & 14 & 18 & 13 \\
\hline - Compensated & 27 & 21 & 48 & 36 & 27 & 26 \\
\hline - Decompensated & 59 & 63 & 48 & 50 & 55 & 61 \\
\hline \multicolumn{7}{|l|}{ Child Pugh class (\%) } \\
\hline$-A$ & 27 & 27 & 20 & 41 & 29 & 26 \\
\hline$-B$ & 33 & 37 & 23 & 14 & 33 & 32 \\
\hline$-C$ & 8 & 9 & 3 & 9 & 5 & 9 \\
\hline - Unknown & 33 & 27 & 55 & 36 & 33 & 32 \\
\hline \multicolumn{7}{|l|}{ Fibrosis stage (\%) } \\
\hline$-<F 3$ & 9 & 9 & 5 & 14 & 9 & 9 \\
\hline$-F 3-F 4$ & 82 & 83 & 73 & 82 & 82 & 81 \\
\hline - Unknown & 10 & 8 & 23 & 5 & 9 & 10 \\
\hline \multicolumn{7}{|l|}{ Hepatocellular carcinoma (\%) } \\
\hline - Never & 58 & 56 & 65 & 68 & 61 & 57 \\
\hline - Past & 17 & 19 & 13 & 14 & 18 & 17 \\
\hline - Current & 12 & 13 & 10 & 9 & 15 & 12 \\
\hline - Unknown & 12 & 12 & 13 & 9 & 6 & 14 \\
\hline \multicolumn{7}{|l|}{ Liver transplantation (\%) } \\
\hline - Never & 34 & 31 & 50 & 32 & 27 & 36 \\
\hline - On list & 13 & 14 & 13 & 9 & 11 & 14 \\
\hline - Before current treatment & 40 & 40 & 33 & 50 & 44 & 38 \\
\hline - During current treatment & 11 & 13 & 3 & 9 & 12 & 10 \\
\hline - Unknown & 2 & 3 & 3 & 0 & 6 & 1 \\
\hline
\end{tabular}

${ }^{\mathrm{a} G e n o t y p e s} 1(n=14), 1 \mathrm{a}(n=58), 1 \mathrm{a} / \mathrm{b}(n=3)$, and 1b $(n=112)$

${ }^{\mathrm{b}}$ Genotypes $3(n=18), 3 \mathrm{a}(n=21)$ and $3 \mathrm{~h}(n=1)$

'Genotypes $2(n=4), 4(n=17)$ and $5(n=1)$

additional covariate for ribavirin use gives a posterior OR for this covariate of 2.36 ( $95 \%$ CI 0.86 to 5.42 ).

The model can then be used to estimate effectiveness in other covariate subgroups rather than in reference patients. Our results suggest a higher rate of SVR in NPP patients without decompensated cirrhosis. For patients with $\mathrm{HCV}$ genotype 1, previously treated but with stable or no cirrhosis, the estimated rate of SVR12 was 97\% (95\% CI 93 to 99\%) and 99\% (95\% CI 96 to 100\%) in intent-to-treat and as-treated analyses respectively. Our results also suggest that treatment might be more effective in NPP patients if it includes ribavirin. For patients with $\mathrm{HCV}$ genotype 1, previously treated and now with decompensated cirrhosis, the estimated rate of SVR12 in an intent-to-treat analysis was $92 \%$ (95\% CI 81 to $98 \%$ ) and $85 \%$ (95\% CI 73 to $93 \%$ ) with and without ribavirin respectively.

\section{Discussion}

This study shows that 24 weeks of daclatasvir and sofosbuvir is an effective treatment for $\mathrm{HCV}$ in patients with advanced liver disease, with estimated rates of SVR12 of $87 \%$ (95\% CI 75 to $94 \%$ ) and $97 \%$ (95\% CI 89 to $100 \%$ ) in intent-to-treat and as-treated analyses respectively. The data are consistent with our expectation that it is easier to treat patients with no or stable cirrhosis because our posterior OR (6.73, 95\% CI 2.42 to 16.1$)$ suggests that treatment at an early stage of infection is even more 
Table 2 Observed sustained virological response rates at 4 weeks (SVR4) and at 12 weeks (SVR12) after completing therapy with daclatasvir and sofosbuvir, with or without ribavirin

\begin{tabular}{|c|c|c|c|c|c|c|c|c|}
\hline \multirow{3}{*}{$\begin{array}{l}\text { Observed } \\
\text { Country }\end{array}$} & \multicolumn{4}{|c|}{ SVR4 } & \multicolumn{4}{|c|}{ SVR12 } \\
\hline & \multicolumn{2}{|c|}{ As-treated } & \multicolumn{2}{|c|}{ Intent-to-treat } & \multicolumn{2}{|c|}{ As-treated } & \multicolumn{2}{|c|}{ Intent-to-treat } \\
\hline & $\%$ & Fraction & $\%$ & Fraction & $\%$ & Fraction & $\%$ & Fraction \\
\hline Austria & 100 & $14 / 14$ & 100 & $14 / 14$ & 100 & $13 / 13$ & 93 & $13 / 14$ \\
\hline Denmark & 94 & $17 / 18$ & 89 & $17 / 19$ & 100 & $17 / 17$ & 89 & $17 / 19$ \\
\hline Spain & 99 & $141 / 142$ & 94 & $141 / 150$ & 99 & $138 / 140$ & 92 & $138 / 150$ \\
\hline Sweden & 100 & $15 / 15$ & 94 & $15 / 16$ & 100 & $15 / 15$ & 94 & $15 / 16$ \\
\hline Switzerland & 85 & $23 / 27$ & 82 & $23 / 28$ & 85 & $22 / 26$ & 85 & $22 / 26$ \\
\hline UK & 100 & $14 / 14$ & 82 & $14 / 17$ & 100 & $14 / 14$ & 82 & $14 / 17$ \\
\hline Overall & 97 & $224 / 230$ & 92 & $224 / 244$ & 97 & $219 / 225$ & 90 & $219 / 242$ \\
\hline
\end{tabular}

beneficial than we anticipated (prior OR 2.0, 95\% CI 0.5 to 8.0). The data do not support our expectation that it is easier to treat naive patients, because our posterior OR $(0.92,95 \%$ CI 0.39 to 1.89$)$ suggests that treatment naivety is less beneficial than we anticipated (prior OR 1.5, $95 \%$ CI 0.38 to 6.0 ). Unfortunately there is little information in these data on how the rate of SVR varies with genotype: posterior ORs for genotype 3 and for other genotypes relative to genotype 1 (OR 0.69, 95\% CI 0.25 to 1.54 , and OR $1.06,95 \%$ CI 0.32 to 2.77 , respectively) are not materially different from our prior ORs (both $1.0,95 \%$ CI 0.25 to 4.0 ). However since the intervals of these posterior ORs are contained within the interval of the prior OR, the data are consistent with our expectation that the rate of SVR does not vary markedly with genotype although the posterior OR for genotype 3 suggests this genotype could be harder to treat. Had we used an alternative prior for genotype 3

Table 3 Observed sustained virological response rates in subgroups at 12 weeks (SVR12) after completing therapy with daclatasvir and sofosbuvir, with or without ribavirin

\begin{tabular}{|c|c|c|c|c|c|}
\hline \multirow[t]{3}{*}{ Subgroups } & & \multicolumn{4}{|c|}{ SVR12 } \\
\hline & & \multicolumn{2}{|c|}{ As-treated } & \multicolumn{2}{|c|}{ Intent-to-treat } \\
\hline & & $\%$ & Fraction & $\%$ & Fraction \\
\hline \multirow[t]{3}{*}{ Genotype $^{a}$} & 1 & 98 & $171 / 174$ & 92 & $171 / 186$ \\
\hline & 3 & 94 & $33 / 35$ & 85 & $33 / 39$ \\
\hline & Other & 94 & $15 / 16$ & 88 & $15 / 17$ \\
\hline \multirow[t]{2}{*}{ Cirrhosis } & None or compensated & 100 & $93 / 93$ & 99 & $93 / 94$ \\
\hline & Decompensated & 95 & $126 / 132$ & 85 & $126 / 148$ \\
\hline \multirow[t]{2}{*}{ Prior treatment } & Naive & 99 & $75 / 76$ & 88 & $75 / 85$ \\
\hline & Experienced & 99 & $144 / 149$ & 92 & $144 / 157$ \\
\hline \multirow[t]{2}{*}{ Ribavirin } & Without & 96 & $157 / 163$ & 88 & $157 / 178$ \\
\hline & With & 100 & $62 / 62$ & 97 & $62 / 64$ \\
\hline Overall & & 97 & $219 / 225$ & 90 & $219 / 242$ \\
\hline
\end{tabular}

${ }^{\mathrm{a}}$ Genotype for the 6 patients without a sustained virologic response 12 weeks after the end of treatment: $1 \mathrm{a}, 1 \mathrm{~b}(n=2), 3 \mathrm{a}(n=2)$ and $5 \mathrm{a}$ reflecting an expectation that genotype 3 would be harder to treat (prior OR 0.67, 95\% CI 0.17 to 2.7 ), we would have found weak support in the data for this position (posterior OR 0.52, 95\% CI 0.20 to 1.14 )

In an additional analysis, we extended our model to include a covariate for ribavirin use. The shift in OR for this covariate (prior OR 1.0, 95\% CI 0.25 to 4.0 ; posterior OR 2.36, $95 \%$ CI 0.86 to 5.42 ), suggests ribavirin use might be beneficial in these patients. Patients that received ribavirin do not seem materially different from those that did not (Table 1) and both groups were treated for a similar duration. The estimated rate of SVR12 in an intent-to-treat analysis was 92\% (95\% CI 81 to 98\%) and 85\% (95\% CI 73 to $93 \%$ ) with and without ribavirin respectively. Other studies suggest that adding ribavirin to this combination is beneficial in patients with decompensated cirrhosis $[20,21]$ but unnecessary when treating patients without cirrhosis [8]; a pattern seen in other oral interferon-free treatment combinations [19].

The strengths of this study include data collected in clinical practice and from patients with advanced liver

Table 4 Estimates from a hierarchical Bayesian model of the sustained virological response rates at 4 weeks (SVR4) and at 12 weeks (SVR12) after completing therapy with daclatasvir and sofosbuvir, with or without ribavirin

\begin{tabular}{|c|c|c|c|c|c|c|c|c|}
\hline \multirow{3}{*}{$\begin{array}{l}\text { Estimate } \\
\text { Country }\end{array}$} & \multicolumn{4}{|c|}{ SVR4 } & \multicolumn{4}{|c|}{ SVR12 } \\
\hline & \multicolumn{2}{|c|}{ As-treated } & \multicolumn{2}{|c|}{ Intent-to-treat } & \multicolumn{2}{|c|}{ As-treated } & \multicolumn{2}{|c|}{ Intent-to-treat } \\
\hline & $\%$ & $95 \% \mathrm{Cl}$ & $\%$ & $95 \% \mathrm{Cl}$ & $\%$ & $95 \% \mathrm{Cl}$ & $\%$ & $95 \% \mathrm{Cl}$ \\
\hline Austria & 97 & $87-100$ & 92 & $81-99$ & 98 & $89-100$ & 88 & $75-96$ \\
\hline Denmark & 93 & $77-100$ & 88 & $73-97$ & 98 & 89-100 & 87 & $73-95$ \\
\hline Spain & 98 & $95-100$ & 92 & $95-100$ & 98 & $94-100$ & 89 & $83-94$ \\
\hline Sweden & 96 & $79-100$ & 90 & $73-100$ & 97 & $85-100$ & 87 & $72-96$ \\
\hline Switzerland & 82 & $56-97$ & 82 & 59-94 & 82 & $57-97$ & 84 & $64-93$ \\
\hline UK & 96 & $80-100$ & 82 & $52-95$ & 97 & $83-100$ & 82 & $56-93$ \\
\hline Overall & 96 & $86-100$ & 89 & $76-96$ & 97 & 89-100 & 87 & 75-94 \\
\hline
\end{tabular}

These estimates apply to patients with genotype 1 hepatitis $C$, previously treated and now with decompensated cirrhosis 
disease including many patients with decompensated cirrhosis (59\%) that previously were difficult to treat. Our data come from a number of European countries and estimates have been appropriately averaged over different health care systems. The limitations include a higher level of missing supporting data than would be expected in a controlled trial. Most patients in this study were infected with $\mathrm{HCV}$ genotype 1 , so that we cannot reliably estimate the effectiveness of this treatment combination in other genotypes or draw conclusions about whether effectiveness differs between genotypes. And most countries contributed only a small number of patients, so that we needed to use advanced statistical methods to sensibly combine data but then more effort is required to interpret our results.

The results of this study are consistent with results from recently completed Phase 2 and 3 studies and with recently published or preliminary results from other early access programmes. In recent Phase 2 and 3 studies, the treatment combination of daclatasvir and sofosbuvir achieved a rate of SVR12 above 95\% in genotype 1 patients without cirrhosis [8]; rates above $95 \%$ in both naive and previously treated genotype 1 patients co-infected with HIV when treated for 12 weeks [22], and a rate above $95 \%$ in genotype 3 patients without cirrhosis [23]. A number of Phase 3 studies of daclatasvir and sofosbuvir (with or without ribavirin) have included at least some patients with cirrhosis and results suggest that lower rates of SVR12 are to be expected: $63 \%$ of 32 genotype 3 patients with compensated cirrhosis achieved a SVR12 after 12 weeks of treatment (without ribavirin) [23]; 86\% of 36 genotype 3 patients with compensated cirrhosis achieved a SVR12 after 12 or 16 weeks of treatment (with ribavirin) [21]; and $79 \%$ of 34 genotype 1 patients with decompensated cirrhosis (Child-Pugh class B or C) achieved a SVR12 after 12 weeks of treatment (with ribavirin) [24]. Data from other early access programmes suggest that this treatment combination is just as effective after liver transplantation $[25,26]$ but effectiveness is reduced in patients with $\mathrm{HCV}$ genotype 3 or decompensated cirrhosis [20, 26, 27].

\section{Conclusions}

Patients with decompensated cirrhosis have always been difficult to treat $[1,28]$. With direct acting antivirals, efficacy is reduced in such patients and it is not yet clear which treatments are best [28-30]. For the moment, the recommended treatment is sofosbuvir in combination with either daclatasvir, ledipasvir or velpatasvir [11, 12] but such recommendations are frequently updated. This study provides evidence that the combination of daclatasvir and sofosbuvir is an effective treatment in clinical practice for HCV genotype 1 patients with decompensated cirrhosis. The study adds to limited evidence that, for these patients, treatment is more effective if ribavirin is added to the combination [20,21]. Other questions remain: whether fewer than 24 weeks of treatment erodes effectiveness in these patients - and by how much [20, 21, 23, 24, 27]; whether this treatment is as effective in HCV genotype 3 patients with decompensated cirrhosis [20,21, 23, 26]; and whether the combination of daclatasvir and sofosbuvir is more effective than other treatment combinations for patients with decompensated cirrhosis [20].

\section{Additional file}

Additional file 1: Tables S1 to S4. This file consists of a title page, a table of contents, a brief introduction to hierarchical Bayesian modelling, references and four tables of results (one table per page). (DOC $90 \mathrm{~kb}$ )

\section{Abbreviations}

95\% Cl: 95\% credible interval; BMS: Bristol-Myers Squibb; HCV: Hepatitis C virus; NPP: Named Patient Programme; OR: Odds ratio; SVR: Sustained virologic response; SVR12: Sustained virologic response at 12 weeks after the end of treatment; SVR4: Sustained virologic response at four weeks after the end of treatment

\section{Acknowledgements}

The authors thank the patients and clinicians who contributed data to this study including: Jose Luis Montero (Hospital Universitario Reina Sofia, Cordoba), Inmaculada Fernandez (Hospital Universitario 12 de Octubre, Madrid), Javier Crespo (Hospital Universitario Marques de Valdecilla, Santander), Agustin Albillos (Hospital Universitario Ramon y Cajal, Madrid), Javier Garcia Samaniego (Hospital Universitario La Paz, Madrid), Javier Ampuero (Virgen del Rocío University Hospital, Sevilla), Carlos Fernandez Carillo (Hospital U Puerta de Hierro, Madrid), John F Dillon (Ninewells Hospital, Dundee), Matthew Priest (Gartnavel General Hospital, Glasgow),Stephen Barclay (Glasgow Royal Infirmary, Glasgow), Andrew Fraser (Aberdeen Royal Infirmary, Aberdeen), David Mutimer (Queen Elizabeth Hospital, Birmingham), Clifford Leen (Western General Hospital, Edinburgh), Matthew Cramp (Derriford Hospital, Plymouth), Birgit Roege Thorup (Kolding Hospital, Kolding), Alex Lund Laursen (Arhus University Hospital, Skejby), Jesper Bach Hansen (Aalborg University Hospital, Aalborg), Mette Kjaer (Copenhagen University Hospital, Rigshospitalet), Mette Rye Clausen (Copenhagen University Hospital, Rigshospitalet) Peer Brehm Christensen (Odense University Hospital, Odense),Francesco Negro (Hôpitaux Universitaires de Genève, Genève), Robert Schvarcz (Karolinska Institutet, Stockholm), Soo Aleman (Karolinska Institutet, Stockholm), Karin Kozbial (Medical University of Vienna, Vienna).

\section{Funding}

This work was supported by BMS. BMS had the right to comment on but not to change the conclusions or content of any publication.

\section{Availability of data and materials}

Individual patient data cannot be shared for contractual reasons. However the first and third hierarchical Bayesian models - models without covariates - can be fit using just the summary data presented in Table 2 . The results of fitting these models are reported in Additional file 1: Tables S1 and S3.

\section{Authors' contributions \\ Study concept and design-DE, HCB, CL, JY; acquisition of data-NW, HH, WI, OW, EG, JMP, LC, MP, JLC; statistical analysis-JY; interpretation of data-JY, NW, WI, OW, RP, CL, DE, HCB; drafting of the manuscript-JY, RP, $\mathrm{CL}, \mathrm{HCB}$; critical revision of the manuscript for important intellectual content-NW, HH, WI, OW, EG, JMP, LC, MP, DE, JLC; administrative support-RP, CL. All authors read and approved the final manuscript.}

\section{Competing interests}

$\mathrm{HCB}$ and $\mathrm{JY}$ - their institution received funding for analysis and writing a manuscript for this study; $\mathrm{HCB}$ - received consulting honorarium from BMS; $J Y$ - no further conflict. JLC received consulting honoraria and speaker fees from BMS, Abbvie and Gilead Sciences. WI received speaker and consultancy 
fees from Roche, Janssen Cilag and Novartis, educational grants from Boehringer Ingelheim, Merck Sharp and Dohme and Gilead Sciences, and research grant support from GlaxoSmithKline, Pfizer, Gilead Sciences and Janssen Cilag; HCV Research UK has received support from Gilead Sciences, BMS, Janssen Cilag, and Abbvie. $\mathrm{HH}$ received lecture fees from AbbVie, Merck Sharp and Dohme, BMS, Gilead Sciences, and Janssen Cilag; advisory board member of AbbVie, Merck Sharp and Dohme, BMS, Gilead Sciences, and Janssen; unrestricted research grant from AbbVie. NW has been clinical investigator for Abbvie, BMS and Merck Sharp and Dohme, and teaching and consulting for Abbvie, BMS, Merck Sharp and Dohme and Gilead Sciences. OW received consultancy fees from AbbVie, BMS, Gilead Sciences, Janssen Cilag, Medivir, Roche and Merck Sharp and Dohme. All other authors - no competing interests.

\section{Consent for publication}

Not applicable.

\section{Ethics approval and consent to participate}

All HCV registries or databases used in this study contained data prospectively collected from adult patients who had given informed consent. These existing data were transferred to the Basel Institute for Clinical Epidemiology and Biostatistics excluding any information that might allow individuals to be identified. As this study used existing anonymous data, no formal ethics approval was required [31].

\section{Author details}

${ }^{1}$ Basel Institute for Clinical Epidemiology and Biostatistics, University Hospital Basel, Spitalstrasse 12, CH-4031 Basel, Switzerland. ²Department of Infectious Diseases, Copenhagen University Hospital, Hvidovre, Denmark. ${ }^{3}$ Department of Internal Medicine III, Division of Gastroenterology and Hepatology, Medical University of Vienna, Vienna, Austria. ${ }^{4} \mathrm{NIHR}$ Nottingham Digestive Diseases Biomedical Research Unit, University of Nottingham, Nottingham, UK. ${ }^{5}$ Division of Infectious Diseases, Department of Medicine, Karolinska Institutet, Karolinska University Hospital Huddinge, Stockholm, Sweden. ${ }^{6}$ Service de Gastroentérologie et Hépatologie, Hôpitaux universitaires de Genève, Geneva, Switzerland. ${ }^{7}$ Hospital Universitario Virgen del Rocío, Seville, Spain. ${ }^{8}$ Liver Unit, Internal Medicine Department, Hospital Universitari Vall Hebron, Barcelona, Spain. ${ }^{9}$ Hepatology Unit, Hospital Universitario y Politécnico La Fe, Valencia, Spain. ${ }^{10}$ Worldwide Health Economics and Outcomes Research, Bristol-Myers Squibb, Uxbridge, United Kingdom. ${ }^{11}$ Worldwide Health Economics and Outcomes Research, Bristol-Myers Squibb, Rueil-Malmaison, France. ${ }^{12}$ Liver Unit, Hospital Universitario Puerta de Hierro, Universidad Autonoma de Madrid, Madrid, Spain.

\section{Received: 28 May 2016 Accepted: 10 December 2016}

\section{Published online: 07 January 2017}

\section{References}

1. Webster DP, Klenerman P, Dusheiko GM. Hepatitis C. Lancet. 2015:385:1124-35.

2. Razavi H, Waked I, Sarrazin C, Myers RP, Idilman R, Calinas F, et al. The present and future disease burden of hepatitis $\mathrm{C}$ virus $(\mathrm{HCV})$ infection with today's treatment paradigm. J Viral Hepat. 2014;21 Suppl 1:34-59.

3. Razavi H, Elkhoury AC, Elbasha E, Estes C, Pasini K, Poynard T, et al. Chronic hepatitis $\mathrm{C}$ virus (HCV) disease burden and cost in the United States. Hepatology. 2013;57:2164-70

4. EASL. Clinical Practice Guidelines: management of hepatitis C virus infection. J Hepatol. 2011;55:245-64.

5. Antaki N, Craxi A, Kamal S, Moucari R, Van der MS, Haffar S, et al. The neglected hepatitis $C$ virus genotypes 4, 5 and 6: an international consensus report. Liver Int. 2010;30:342-55.

6. Bunchorntavakul C, Reddy KR. Review article: the efficacy and safety of daclatasvir in the treatment of chronic hepatitis $C$ virus infection. Aliment Pharmacol Ther. 2015;42:258-72.

7. McCormack PL, Lyseng-Williamson KA. Daclatasvir in hepatitic C virus infection: a guide to its use in the EU. Drugs Ther Perspect. 2016;32:42-9.

8. Sulkowski MS, Gardiner DF, Rodriguez-Torres M, Reddy KR, Hassanein T, Jacobson I, et al. Daclatasvir plus sofosbuvir for previously treated or untreated chronic HCV infection. N Engl J Med. 2014;370:211-21.

9. Asselah T. Daclatasvir plus sofosbuvir for HCV infection: an oral combination therapy with high antiviral efficacy. J Hepatol. 2014;61:435-8.

10. Hsu CS, Kao JH. Management of hepatitis C patients with decompensated liver disease. Expert Rev Gastroenterol Hepatol. 2016;10:679-88.
11. AASLD-IDSA. Recommendations for testing, managing, and treating hepatitis C. 2016. http://www.hcvguidelines.org/full-report/unique-patientpopulations-patients-decompensated-cirrhosis. Accessed 11 Nov 2016.

12. EASL. Recommendations on treatment of hepatitis C 2016. $2016 \mathrm{http}: / /$ www.easl.eu/research/our-contributions/clinical-practice-guidelines/detail/ easl-recommendations-on-treatment-of-hepatitis-c-2016. Accessed 11 Nov 2016.

13. Gelman A, Carlin JB, Stern HS, Dunson DB, Vehtari A, Rubin DB. third. Bayesian data analysis. Boca Raton: CRC Press; 2013.

14. Greenland S. Prior data for non-normal priors. Stat Med. 2007;26:3578-90.

15. Greenland S. Bayesian perspectives for epidemiological research: I. Foundations and basic methods. Int J Epidemiol. 2006;35:765-75.

16. Greenland S. Bayesian perspectives for epidemiological research. II. Regression analysis. Int J Epidemiol. 2007;36:195-202.

17. Young J, Scherrer AU, Gunthard HF, Opravil M, Yerly S, Boni J, et al. Efficacy, tolerability and risk factors for virological failure of darunavir-based therapy for treatment-experienced HIV-infected patients: the Swiss HIV Cohort Study. HIV Med. 2011;12:299-307.

18. Wedemeyer $\mathrm{H}$. Towards interferon-free treatment for all HCV genotypes. Lancet. 2015;385:2443-5.

19. Hezode C, Bronowicki JP. Ideal oral combinations to eradicate HCV: the role of ribavirin. J Hepatol. 2016:64:215-25.

20. Foster GR, Irving WL, Cheung MC, Walker AJ, Hudson BE, Verma S, et al. Impact of direct acting antiviral therapy in patients with chronic hepatitis C and decompensated cirrhosis. J Hepatol. 2016;64:1224-31.

21. Leroy V, Angus P, Bronowicki JP, Dore GJ, Hezode C, Pianko S, et al. Daclatasvir, sofosbuvir, and ribavirin for hepatitis $C$ virus genotype 3 and advanced liver disease: a randomized Phase III study (ALLY-3+). Hepatology. 2016; 63:1430-41.

22. Wyles DL, Ruane PJ, Sulkowski MS, Dieterich D, Luetkemeyer A, Morgan TR, et al. Daclatasvir plus sofosbuvir for HCV in patients coinfected with HIV-1. N Engl J Med. 2015;373:714-25.

23. Nelson DR, Cooper JN, Lalezari JP, Lawitz E, Pockros PJ, Gitlin N, et al. Alloral 12-week treatment with daclatasvir plus sofosbuvir in patients with hepatitis C virus genotype 3 infection: ALLY-3 phase III study. Hepatology. 2015;61:1127-35.

24. Poordad F, Schiff ER, Vierling JM, Landis C, Fontana RJ, Yang R, et al. Daclatasvir with sofosbuvir and ribavirin for HCV infection with advanced cirrhosis or post-liver transplant recurrence. Hepatology. 2016;63:1493-505.

25. Coilly A, Fougerou-Leurent C, de Ledinghen V, Houssel-Debry P, Duvoux C, Di Martino $\mathrm{V}$, et al. Multicentre experience using daclatasvir and sofosbuvir to treat hepatitis C recurrence - The ANRS CUPILT study. J Hepatol. 2016;65:711-8.

26. Welzel TM, Petersen J, Herzer K, Ferenci P, Gschwantler M, Wedemeyer H, et al. Daclatasvir plus sofosbuvir, with or without ribavirin, achieved high sustained virological response rates in patients with HCV infection and advanced liver disease in a real-world cohort. Gut. 2016;65:1861-70.

27. Hezode C, De Ledinghen V, Fontaine H, Zoulim F, Lebray P, Boyer N, et al. Daclatasvir plus sofosbuvir with or without ribavirin in patients with HCV genotype 3 infection: interim analysis of a French multicenter compassionate use program (abstract LP05). J Hepatol. 2015;62:S265-6.

28. Nakamoto S, Kanda T, Shirasawa H, Yokosuka O. Antiviral therapies for chronic hepatitis C virus infection with cirrhosis. World J Hepatol. 2015;7:1133-41.

29. Boccaccio V, Bruno S. Management of HCV patients with cirrhosis with direct acting antivirals. Liver Int. 2014;34 Suppl 1:38-45.

30. Gambato M, Lens S, Navasa M, Forns X. Treatment options in patients with decompensated cirrhosis, pre- and post-transplantation. J Hepatol. 2014;61: S120-31.

31. CIOMS. International ethical guidelines for epidemiological studies. 2009 http://www.cioms.ch/index.php/publications/printablev3/541/ showCategory/65/bioethics-and-health-policy-guidelines-and-othernormative-documents. Accessed 9 Dec 2016. 